\title{
Surface modification of silica with a hydrophilic polymer and its influence on reinforcement of natural rubber latex
}

\author{
M.C.W. Somaratne ${ }^{1 *}$, N.M.V.K. Liyanage ${ }^{2}$ and S. Walpalage ${ }^{3}$ \\ ${ }^{1}$ Institute of Technology, University of Moratuwa, Katubedda, Moratuwa. \\ ${ }^{2}$ Department of Materials Science and Engineering, Faculty of Engineering, University of Moratuwa, Katubedda, Moratuwa. \\ ${ }^{3}$ Department of Chemical and Process Engineering, Faculty of Engineering, University of Moratuwa, Katubedda, Moratuwa.
}

\begin{abstract}
Surface modification of precipitated silica particles was carried out in both aqueous and non aqueous media separately with a polyacrylic based hydrophilic polymer synthesized in the laboratory. FTIR spectroscopy and thermo gravimetric analysis of the modified and unmodified silica particles were performed to confirm successful surface modification. Colloidal stability of the aqueous dispersions of the unmodified/modified silica particles were observed at different $\mathrm{pH}$ levels. The dispersions at neutral $\mathrm{pH}$ were incorporated in different proportions to un-compounded and compounded natural rubber latex (NRL) separately and thin latex films were produced from them by casting. Dispersability of silica particles within the rubber matrix was examined through microstructure studies of films cast from unmodified/modified silica incorporated un-compounded NRL. The influence of surface modification upon the reinforcement of NRL was investigated through tensile and tear strength properties of vulcanized NRL cast films containing modified silica. The uniform dispersion of silica particles and the improved mechanical properties envisaged better compatibility of modified silica particles with NRL by means of interfacial interactions. A significant improvement in the mechanical properties was observed in the films produced with $5-7$ phr of modified silica.
\end{abstract}

Keywords: Hydrophilic polymers, silica, surface modification, tear strength, tensile strength, vulcanized NRL.

\section{INTRODUCTION}

Direct reinforcement of latex is one of the most long standing problems faced by latex products manufacturing industries, especially in natural rubber latex (NRL) based processes. Literature available on the reinforcement of NRL compounds is very limited and there is no established practical commercial scale method available for the reinforcement of NRL films, although it is very well established for dry rubber processes.

Some researchers have reported that methylmethacrylate grafted natural rubber latex provides direct reinforcement and increases tear and puncture resistance significantly, whilst some polymeric materials such as terpolymers of vinyl acetate/vinyl chloride/ ethylene, copolymers of vinyl acetate/ethylene and polystyrenes have been reported as organic reinforcing fillers for latex (Cai et al., 2003). In addition, inorganic fillers are also added to latex compounds with the aim of reducing the cost of the latex compound. Most of these fillers act only as diluents and are not capable of imparting any reinforcement. $\mathrm{CaCO}_{3}$ is the common filler of this type, however, it has been reported that fine particles of $\mathrm{CaCO}_{3}$ reinforce natural rubber latex (Cai et al., 2003; Manroshan \& Baharin, 2005; Deng et al., 2006).

The widely used non black reinforcing filler in dry rubber compounds is silica, of which the surface activity is created by surface bound hydroxyl groups (Wanger, 1976). However the hydrophilic nature of these hydroxyl groups reduces the compatibility with hydrophobic rubber and therefore, the particles in dry rubber mixture tend to build filler-filler interactions rather than forming fillerrubber interactions. This effect is even more significant in NRL since the aqueous medium in which the rubber particles are dispersed encourages the self-aggregation of hydrophilic filler particles. Subsequently, the aggregated high dense particles tend to settle down as a separate layer at the bottom of the latex. 
An effective and easily applicable technique, which discourages such aggregations while enhancing rubberfiller interactions is the modification of the surface of silica particles. Several techniques for the surface modification of silica have been reported. Among them silane coupling agents are very popular and they are capable of dispersing silica uniformly in the rubber matrix confirming improved mechanical properties on the dry natural rubber vulcanisate (Anasarifar et al., 2005; Tian et al., 2005; Prasertsri \& Rattanasom, 2011) although the same technique is not applicable for latex. Other methods such as in-situ grafting polymerization (Suzuki et al., 2000; Gultek \& Seckin, 2002; Nguyen et al., 2003; Park et al., 2010), admicellar polymerization (Nontasorn et al., 2005; Rangsunvigit et al., 2008), surface initiated redox polymerization (Salarizadeh et al., 2012) and plasma polymerization (Nah et al., 2002) have been reported for surface modification of silica with polymers. Macromolecular coupling agents (Tai et al., 2008; Xia et al., 2008a; b) have also been used to modify the surface of silica particles.

A considerable amount of research has been carried out on the surface modification of silica intended for a wide range of applications. Among them are the reinforcement of dry rubber and plastic materials $(\mathrm{Ru}$ et al., 2006; Peng et al., 2007; Jeong et al., 2009; Park et al., 2010). However, surface modified silica has not been successfully used for the reinforcement of NRL in large scale latex based product manufacture.

The present study is based on the surface modification of silica with a hydrophilic polymer containing carboxylic acid groups synthesised in the laboratory. The aqueous dispersions of modified silica are incorporated into NRL with the objective of minimizing self aggregations of filler particles, while enhancing the rubber-filler interfacial interactions at latex stage.

\section{METHODS AND MATERIALS}

A concentrated low ammonia NRL sample obtained from the Leefern Laboratory (Pvt.) Ltd. and a sample of silica (Ultrasil VN3) obtained from the Chemical Industries Colombo Ltd. were used throughout this study. All the other chemicals and reagents were obtained from local chemical suppliers.

\section{Synthesis of the hydrophilic polymer}

The hydrophilic polymer was synthesized by following the free radically initiated solution polymerization process. A mixture of $0.4 \mathrm{~g}$ of benzoyl peroxide initiator and $40 \mathrm{~g}$ of methacrylic acid monomer was dissolved in $60 \mathrm{~g}$ of xylene. Another portion of $100 \mathrm{~g}$ of xylene solvent was transferred to a five neck reaction flask and heated up to $90{ }^{\circ} \mathrm{C}$. The monomer, initially dissolved in xylene was added slowly within $30 \mathrm{~min}$ to the xylene in the reaction vessel at $90{ }^{\circ} \mathrm{C}$ while constantly stirring and bubbling nitrogen gas to the sealed vessel. The reaction was allowed to continue for $5 \mathrm{~h}$ under the same conditions and the resultant polymer, which was separated as a precipitate was isolated by vacuum filtration. The polymer was then vacuum-dried at $60^{\circ} \mathrm{C}$ for $10 \mathrm{~h}$ before use.

\section{Measurement of viscosity of diluted solutions of the polymer}

The polymer $(0.5 \mathrm{~g})$ was dissolved in ethanol to make $1 \%$ $(w / w)$ solution and it was then further diluted in ethanol to make a series of solutions i.e. $0.125 \%, 0.25 \%, 0.5 \%$ and $0.75 \%$. The viscosity of the diluted solutions was measured by using an Ubbelohde viscometer (Capillary no. 0 ) at $40{ }^{\circ} \mathrm{C}$. The measurement of viscosity is usually made by comparing the efflux time $t$ required to flow through the capillary tube by a specified volume of the polymer solution with the corresponding efflux time $\mathrm{t}_{0}$ of the solvent.

Relative viscosity $\left(\eta_{\text {rel }}\right)=t / t_{0}$ Specific viscosity $\left(\eta_{\mathrm{sp}}\right)=\left(\mathrm{t}-\mathrm{t}_{0}\right) / \mathrm{t}_{0}$

Reduced viscosity $\left(\eta_{\text {red }}\right)=\eta_{\mathrm{sp}} / \mathrm{c}$

Intrinsic viscosity $(\eta)=\left(\eta_{\mathrm{sp}} / \mathrm{c}\right)_{\mathrm{c}=0}$

where c - concentration of the polymer solution in $\mathrm{g} / 100 \mathrm{~mL}$

Viscosity data as a function of concentration were extrapolated to infinite dilution by means of Huggins equation (Huggins, 1942) as follows.

$\eta_{\mathrm{sp}} / \mathrm{c}=[\eta]+\mathrm{k}^{\prime}[\eta]^{2} \mathrm{c}$

where $\mathrm{k}^{\prime}$ - constant

\section{Surface modification of silica}

Surface modification of silica was performed in both reaction media, the aqueous and the non-aqueous.

\section{Surface modification in non-aqueous medium}

Ten grams of silica powder (Ultrasil VN3) of which the surface area was $175 \mathrm{~N}_{2}\left(\mathrm{~m}^{2} / \mathrm{g}\right)$ and the average particle size was $31 \mu \mathrm{m}$ was mixed with $60 \mathrm{~mL}$ of xylene. A weight of $0.5 \mathrm{~g}$ of the synthesized hydrophilic polymer was also added to the same mixture. The mixture was then heated to $100{ }^{\circ} \mathrm{C}$ and maintained at $100{ }^{\circ} \mathrm{C}$ for $3 \mathrm{~h}$ 
while stirring. The modified filler (MFn) was isolated by filtration and vacuum dried for $10 \mathrm{~h}$. A sample of MFn (1 mg) was washed with $200 \mathrm{~mL}$ of tetrahydrofuran (THF) in a Soxhelt apparatus for $72 \mathrm{~h}$ to remove free polymer chains. The modified/washed filler sample (MFnW) was vacuum-dried and used to confirm the surface modification reaction.

\section{Preparation of aqueous dispersions of unmodified filler and MFn}

Two separate sets of $15 \% \mathrm{w} / \mathrm{w}$ aqueous dispersions of silica i.e. unmodified (UMF) and MFn, were prepared by grinding the requisite chemicals (according to the formulation given in Table 1) in a pot mill for $10 \mathrm{~h}$.

Table 1: Formulation used in the preparation of aqueous dispersions of UMF and MFn

\begin{tabular}{lcc}
\hline Ingredient & \multicolumn{2}{c}{ Weight $(\mathrm{g})$} \\
\hline UMF & 7.5 & - \\
MFn & - & 7.875 \\
Dispersing agent & 0.5 & 0.5 \\
Water & 42.0 & 41.625 \\
Total & 50.0 & 50.0 \\
\hline
\end{tabular}

\section{Surface modification in aqueous medium}

Surface modification of silica in aqueous medium was carried out at room temperature. A weight of $7.5 \mathrm{~g}$ of silica filler and $0.375 \mathrm{~g}$ of hydrophilic polymer powder was mixed with water and a $15 \% \mathrm{w} / \mathrm{w}$ aqueous dispersion of silica (MFa) was prepared according to the formulation given in Table 2, by grinding the ingredients in a pot mill for $10 \mathrm{~h}$

Table 2: Formulation used in the modification of filler in an aqueous medium

\begin{tabular}{lc}
\hline Ingredient & Weight $(\mathrm{g})$ \\
\hline Silica (UMF) & 7.5 \\
Polymer & 0.375 \\
Dispersing agent & 0.5 \\
Water & 41.625 \\
Total & 50.0 \\
\hline
\end{tabular}

A sample of the MFa dispersion was air dried and then vacuum-dried to evaporate the water content. A portion (1 mg) of the dried MFa sample was washed with THF for $72 \mathrm{~h}$ to remove the free and loosely bound polymer chains. The dried $\mathrm{MFa}$ and washed $\mathrm{MFa}(\mathrm{MFaW})$ were characterized to confirm the surface modification reaction.

\section{FTIR studies of surface modified fillers}

Fourier transform infrared (FTIR) spectra of $\mathrm{KBr}$ pellets prepared separately from hydrophilic polymer and filler samples, namely, UMF, MFn, MFa, MFnW and MFaW were obtained using a ALPHA FTIR spectrophotometer provided by Bruker to confirm the chemical and physical interactions between the polymer and silica particles.

\section{Thermo gravimetric analysis (TGA) of modified fillers}

Thermo gravimetric analysis (TGA) of the hydrophilic polymer, UMF, MFn and MFnW was performed by increasing the temperature from room temperature $\left(28+/-2{ }^{\circ} \mathrm{C}\right)$ to $600{ }^{\circ} \mathrm{C}$ at a heating rate of $10{ }^{\circ} \mathrm{C} / \mathrm{min}$ using the rheometric scientific STA 1500 instrument.

\section{Stability studies of aqueous dispersions of fillers}

When unloading the aqueous dispersions of UMF, MFn and $\mathrm{MFa}$ the $\mathrm{pH}$ was within the range of $4.5-6.5$ and it was observed that the filler particles settled to the bottom of the container with time. The stability variations of these dispersions were observed visually at different $\mathrm{pH}$ levels by increasing the $\mathrm{pH}$ up to 7 and 10 of two small portions of each dispersion by adding $15 \% \mathrm{KOH}$ aqueous solution.

\section{Preparation of NRL/modified silica composites}

The aqueous dispersions of UMF, MFa and MFn at neutral $\mathrm{pH}$ were mixed separately with un-compounded and compounded (after adding vulcanizing ingredients) latex at 4 different dosages i.e. $5 \mathrm{phr}, 10 \mathrm{phr}, 15 \mathrm{phr}$ and $20 \mathrm{phr}$ of silica. The filled latex samples were matured for $20 \mathrm{~h}$ and thin films (thickness $<0.5 \mathrm{~mm}$ ) from them were cast on plastic moulds.

\section{Microstructure studies}

Microstructures of the cross-sections of cast NRL unvulcanized films containing $20 \mathrm{phr}$ of unmodified and modified filler were examined through MX 7100 NEIJI optical microscope separately. The films containing $20 \mathrm{phr}$ of silica were used for this observation as the films with UMF separated clearly into two layers of rubber and silica.

\section{Measurement of physical properties}

Tensile and tear strength of the NRL vulcanizates were measured using a Hounsfield H10KT tensile testing machine as per the standard test methods for latex films (ISO 37, 2011 and ISO 34-1, 2010, respectively) at room temperature $\left(28+/-2^{\circ} \mathrm{C}\right)$. 


\section{RESULTS}

Viscosity average molecular weight of synthesized polymer

The intrinsic viscosity of the polymer as obtained from Figure 1 was 0.204 . The viscosity average molecular weight of the hydrophilic polymer was calculated using the Mark-Houwink equation.

$[\eta]=\mathrm{kM}^{\mathrm{a}}$

where

$\mathrm{k}=2.42 \times 10^{-3} \mathrm{~cm}^{3} / \mathrm{g}$

$\mathrm{a}=0.51$, for the solvent ethanol (Mason, 1975)

$[\eta]=0.204$ (from the above graph)

The calculated value of the viscosity average molecular weight of the polymer was 5972 .

\section{FTIR studies}

FTIR spectra of the hydrophilic polymer, UMF, MFa, MFn, MFaW and MFnW are shown in Figure 2.

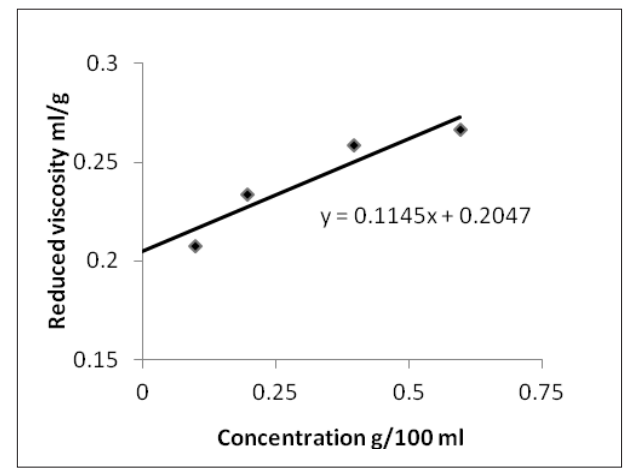

Figure 1: The variation of reduced viscosity against concentration

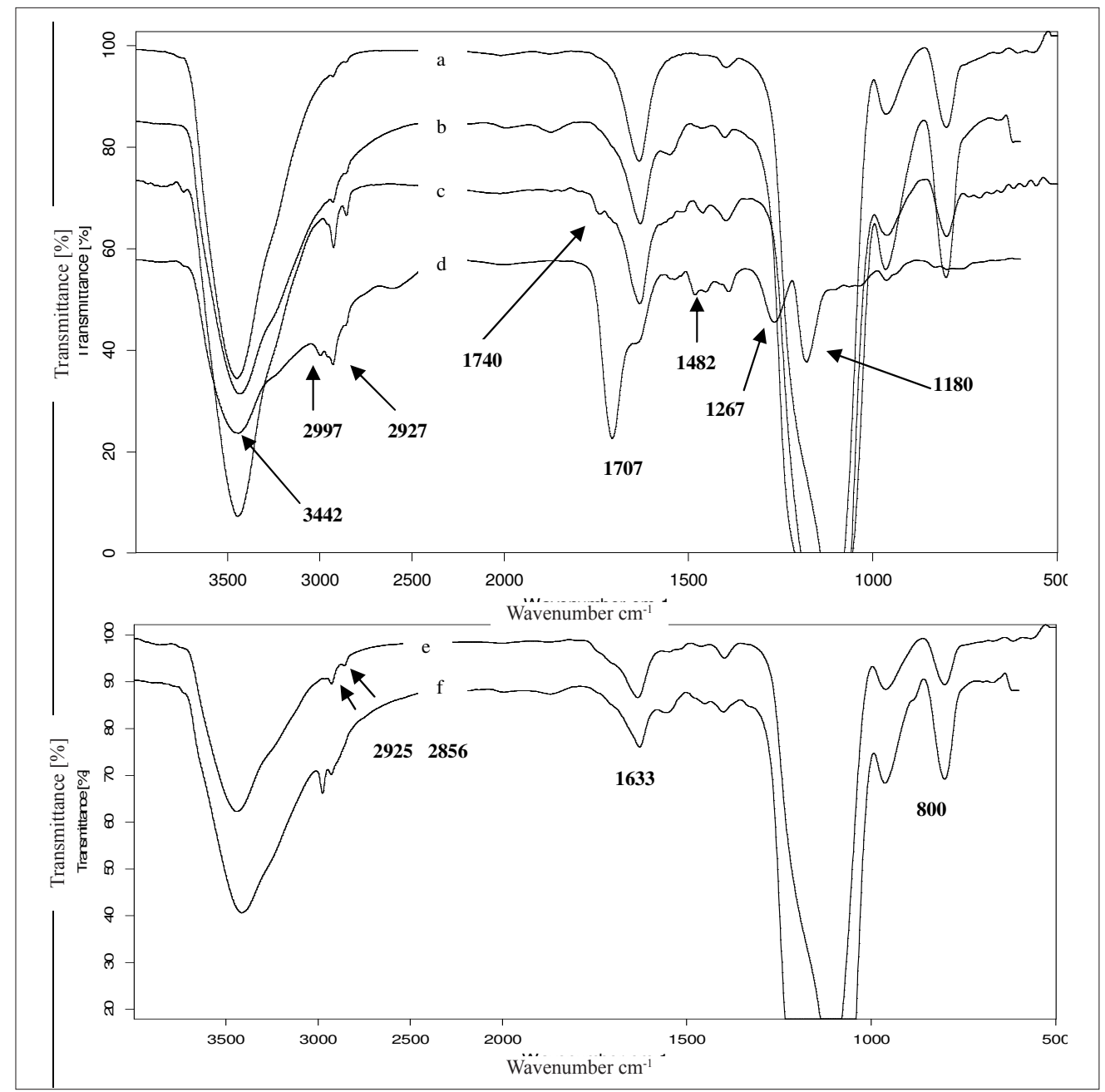

Figure 2: FTIR spectra of (a) UMF; (b) MFaW; (c) MFnW; (d) polymer; (e) MFn and (f) MFa 


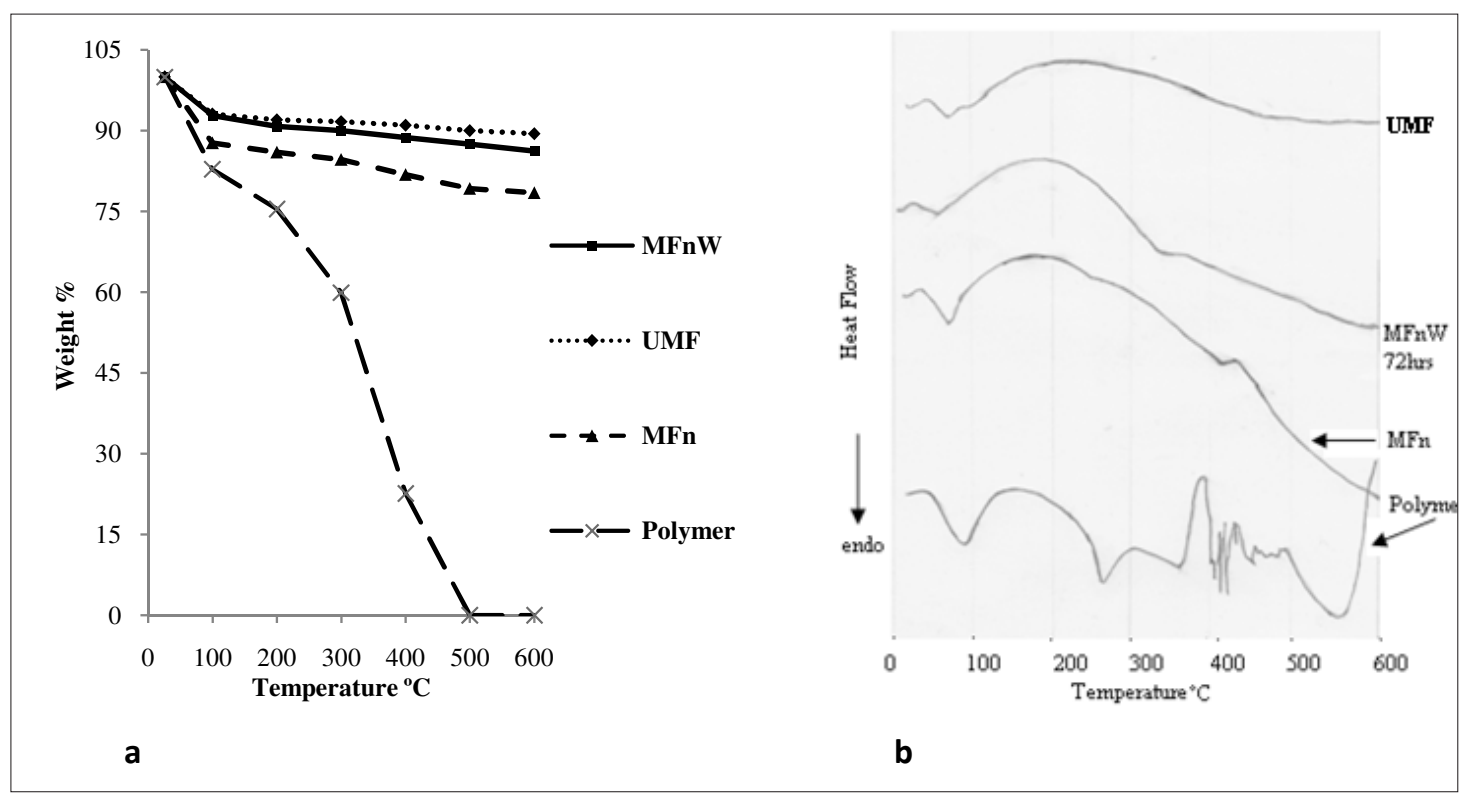

Figure 3: Thermal analysis (a) TGA and (b) DTA of the polymer, UMF, MFn and MFaW

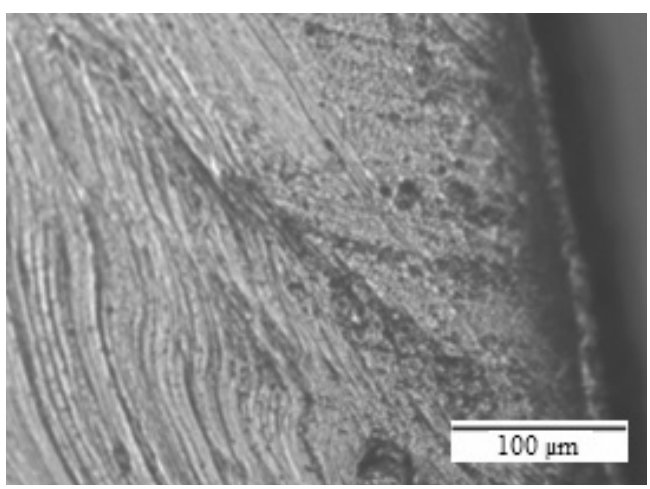

(a)

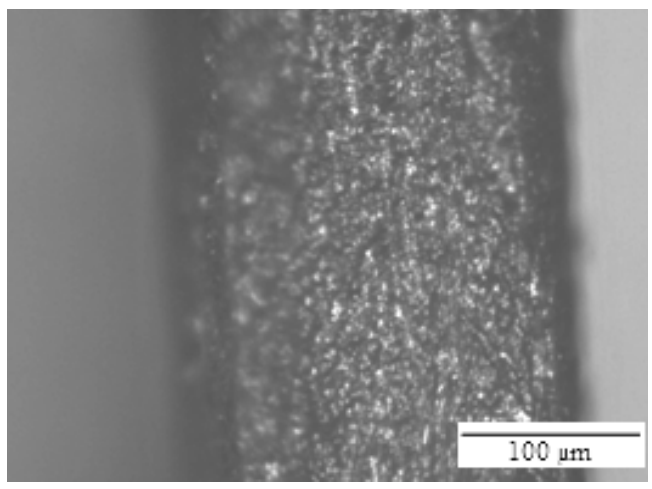

(c)

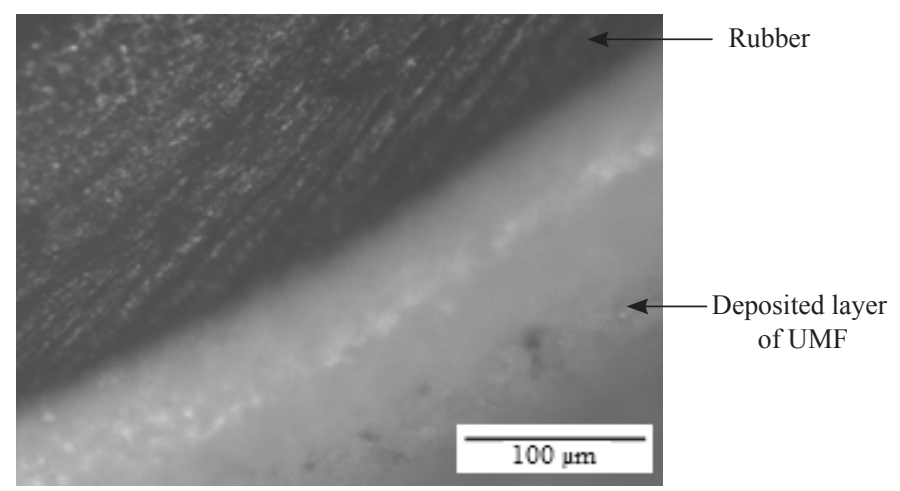

(b)

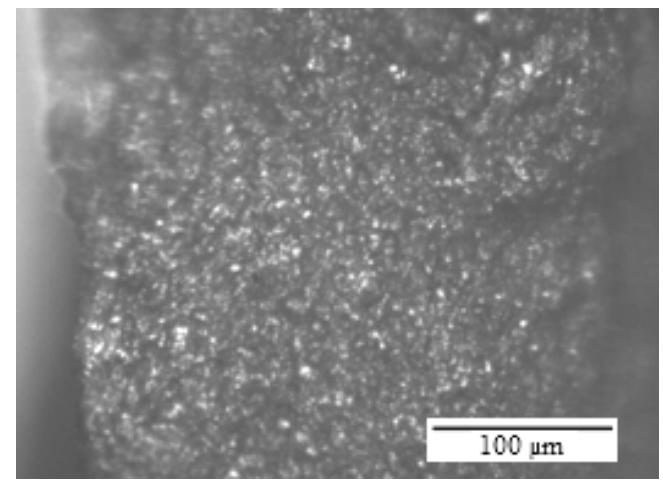

(d)

Figure 4: Microstructures of cross-sections of un-vulcanized latex films (a) unfilled and filled with (b) UMF; (c) MFa and (d) MFn 
Thermo gravimetric analysis (TGA) of modified fillers

The variation of mass loss and differential thermal analysis (DTA) of the polymer, UMF, MFn and MFnW are given in Figure 3 a and b, respectively.

\section{Stability studies of dispersions at different pH levels}

The dispersability of the modified filler dispersions was found to increase with the formation of a high viscous gel as the $\mathrm{pH}$ increased up to 7. However, the stability of dispersion of the MFa was found to reduce with further increase of the $\mathrm{pH}$ whilst that of MFn was maintained even at higher $\mathrm{pH}$ values.

\section{Microstructures}

Microstructures of the cross sections of the cast films of unfilled latex and latex filled with UMF, MFa and MFn are shown in Figure 4. The modified filler (MF) added films show uniform dispersion of filler particles throughout the rubber matrix, while the film containing UMF shows two separate layers as rubber and filler.

\section{Physical properties}

The variation of tear strength and tensile strength with the concentration of UMF and MF are shown in Figure 5 and 6 , respectively.

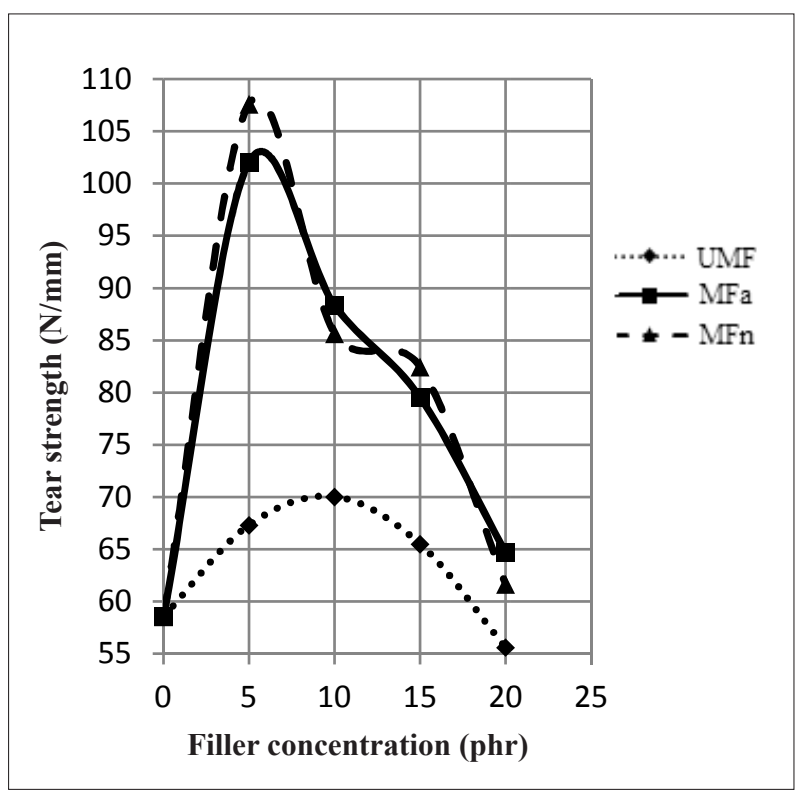

Figure 5: Effect of filler loading on tear strength of vulcanized NRL cast films

\section{DISCUSSION}

The FTIR spectrum in Figure 2 (a) was obtained for unmodified silica filler and its significant broad peak in between 3700 and $3000 \mathrm{~cm}^{-1}$ is attributed to the stretching vibrations of hydrogen bonded hydroxyl groups $(-\mathrm{OH})$ and the medium peak at $1633 \mathrm{~cm}^{-1}$ is assigned to the bending vibrations of - $\mathrm{OH}$ group. The very strong peak at $1106 \mathrm{~cm}^{-1}$ with a shoulder is attributed to $\mathrm{Si}-\mathrm{O}-\mathrm{Si}$ asymmetric stretching vibrations while the peak at $800 \mathrm{~cm}^{-1}$ is assigned for Si-O-Si symmetric stretching vibrations. The peak at $964 \mathrm{~cm}^{-1}$ is attributed to $\mathrm{Si}-\mathrm{OH}$ vibrations (Musići et al., 2011)

The FTIR spectrum of the polymer is shown in Figure 2 (d). The peak corresponding to the stretching vibration of hydrophilic group (carboxylic acid) is observed at $1707 \mathrm{~cm}^{-1}$ and a shoulder peak at $1707 \mathrm{~cm}^{-1}$ to the right is due to the $\mathrm{O}-\mathrm{H}-\mathrm{O}$ bending vibrations of loosely bound water molecules. Asymmetric stretching vibrations of $-\mathrm{CH}_{3}$ and $-\mathrm{CH}_{2}-$ groups are observed at 2997 and $2929 \mathrm{~cm}^{-1}$, respectively and the doublet of weak peaks at $1482 \mathrm{~cm}^{-1}$ is attributed to the bending vibrations of the $-\mathrm{CH}_{3}$ and $-\mathrm{CH}_{2}$ - groups. The significantly broad peak at $3442 \mathrm{~cm}^{-1}$ is attributed to the stretching vibrations of the hydroxyl group. The other two strong peaks at 1267 and $1180 \mathrm{~cm}^{-1}$ are corresponded to the stretching vibrations of -C-C- skeletal and -C-O bond (Coates, 2000).

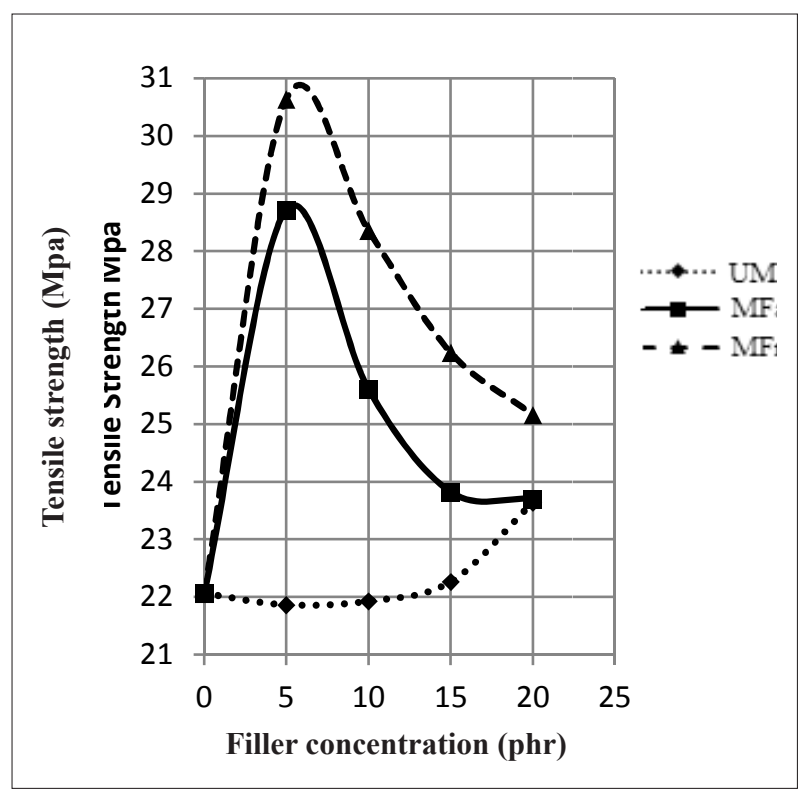

Figure 6: Effect of filler loading on tensile strength of vulcanized NRL cast films 
The FTIR spectrum of the filler modified in non aqueous medium with the hydrophilic polymer (MFn) is shown by the spectrum 2(e). The broad peak at $3442 \mathrm{~cm}^{-1}$ corresponds to the unreacted $-\mathrm{OH}$ groups of both UMF and the polymer. The peaks at $2925 \mathrm{~cm}^{-1}$ and $2856 \mathrm{~cm}^{-1}$ are attributed to the asymmetric stretching vibration of $-\mathrm{C}-\mathrm{H}$ bonds. Polymer chains attached to the surface of silica particles contain $-\mathrm{CH}_{2}$ and $-\mathrm{CH}_{3}$ groups, however the original peaks attributed to such alkyl groups of the polymer at $2997 \mathrm{~cm}^{-1}$ and $2927 \mathrm{~cm}^{-1}$ have shifted to lower wave numbers as 2925 and $2856 \mathrm{~cm}^{-1}$, respectively after the surface modification reaction. The peak attributed to carboxylic acid groups of the polymer at $1707 \mathrm{~cm}^{-1}$ appears as a shoulder from $1740-1705 \mathrm{~cm}^{-1}$ to the peak at $1633 \mathrm{~cm}^{-1}$ of the spectrum of MFn (2e). That is due to the newly formed covalent bond as given in structure (i)<smiles>[CH]=O</smiles>
-C-O-Si-

(i)

between the hydroxyl group of silica and the carboxylic acid of the polymer and/or hydrogen bonds formed between the same groups. Therefore the weakly attached macromolecules were washed out using THF to verify the nature of the newly formed bonds.

The broad peak in the range of $1740-1705 \mathrm{~cm}^{-1}$ corresponding to the hydrogen bonded carboxylic acid groups of the spectrum 2(e) has disappeared by washing with THF, while a new peak becomes visible at $1740 \mathrm{~cm}^{-1}$ as shown in the spectrum 2(c), which corresponds to the MFn washed for $72 \mathrm{~h}$ (MFnW). The new peak at $1740 \mathrm{~cm}^{-1}$ is attributed to the carbonyl ester group of the newly formed covalent bond (structure i) between the polymer and silica filler. It ensures the esterification reaction that occurs between the carboxylic group of the polymer and the silanol group of the silica filler. A similar observation has been encountered by Ru et al. (2006) and other scientists in their studies of surface modification of silica (Wu \& Liao, 2003; Tai et al., 2008; Xia et al., 2008b). Young (1969) also reported the same observation in his study on IR absorption on carboxylic acids and their derivatives adsorbed on silica.

The corresponding peak of the C-O-Si group of the newly formed bond should appear at $1110-1080 \mathrm{~cm}^{-1}$, however in the present observation it is masked by the strong peak attributed to Si-O-Si at $1107 \mathrm{~cm}^{-1}$. Furthermore, the remaining peaks at $2925 \mathrm{~cm}^{-1}$ and $2856 \mathrm{~cm}^{-1}$ of the spectrum of MFnW for 72 hours 2(c), also confirm the covalently bonded polymer chains to the filler surface as those peaks are attributed to the stretching vibration of $-\mathrm{C}-\mathrm{H}$ bonds of the polymer molecule. Therefore, it confirms the formation of covalent bonds through chemical reaction between the carboxylic acid groups of the polymer and the hydroxyl groups of the filler by eliminating a water molecule in non-aqueous medium. However, after washing the MFa for 72 hours a new peak was not seen at $1740 \mathrm{~cm}^{-1}$ in the spectrum of MFaW 2(b). The reason is that the esterification reaction is not viable in aqueous medium towards forward direction because the medium is rich in water molecules.

In addition, there is an option to form hydrogen bonds between the same functional groups of the filler and polymer as shown in Figure 7. The carbonyl groups are capable of forming hydrogen bonds with silica and the hydrogen bonded carbonyl groups show a broader peak in FTIR spectrum (Shing \& Kim, 2001). In the present study broad peaks were observed in the range of $1740-1705 \mathrm{~cm}^{-1}$ of MFn and MFa spectra (e and f) of Figure 2 and it is an evidence to confirm the formation of hydrogen bonds after modification reactions in both reaction media. Therefore, it appears that only hydrogen bonds are present in MFa. Consequently, both types of modified fillers contain newly formed covalent and/or hydrogen bonds between the carboxylic acid groups of the polymer and the hydroxyl groups of the filler.

As shown in Figure 3, all the samples desorb the physically adsorbed water molecules nearly up to $200{ }^{\circ} \mathrm{C}$ and the thermal decomposition of the polymer starts at about $260{ }^{\circ} \mathrm{C}$ followed by a drastic mass loss with increasing temperature. The first stage of thermal transition may be due to anhydride formation between the carboxylic groups and the release of $\mathrm{CO}_{2}$. However, the anhydride formation dominates the minor decarboxylation reaction. The major decomposition is due to the fragmentation of anhydride ring structure with the evolution of $\mathrm{CO}_{2}, \mathrm{CO}$, propene, isobutylene, and different alkenes, alkynes, etc. (Azhgozhinova et al., 2004).

The surface hydroxyl groups of the unmodified silica are subdivided into three types, namely, isolated free silanols, germinal silanols and vicinal or hydrogen bonded silanol. In addition, there are silanol groups with physically adsorbed water. The weight loss up to $190^{\circ} \mathrm{C}$ is attributed to the loss of such physically adsorbed water from the silica surface followed by a broad region of weight loss up to $600{ }^{\circ} \mathrm{C}$, which is due to the dehydroxylation. At this stage the removal of vicinal hydroxyl groups starts, leaving free siloxane groups (Zhuravlev, 2000).

The MFn and MFnW show the combined effect of mass loss attributed to the thermal decompositions of the polymer and UMF. The percentage of mass loss of UMF, MFn and MFnW is given in Table 3. 
Table 3: Mass loss of UMF, MFn washed and MFn unwashed fillers

\begin{tabular}{lrrr}
\hline & UMF & MFn & MFnW \\
\hline Total mass loss $\%$ & 10.59 & 21.54 & 13.80 \\
Mass loss up to $200{ }^{\circ} \mathrm{C}(\%)$ & 8.03 & 14.00 & 9.20 \\
Mass loss from $200{ }^{\circ} \mathrm{C}$ to $600{ }^{\circ} \mathrm{C}(\%)$ & 2.56 & 7.54 & 4.60 \\
Mass loss of polymer from $200{ }^{\circ} \mathrm{C}$ & - & 4.98 & 2.04 \\
to $600{ }^{\circ} \mathrm{C}(\%)$ & & & \\
\hline
\end{tabular}

The desorption of physically adsorbed water of UM silica and polymer were the only thermal transitions that could be seen up to $200{ }^{\circ} \mathrm{C}$. Accordingly the percentage loss of water is determined by calculating the mass loss up to $200{ }^{\circ} \mathrm{C}$. The mass loss from 200 to $600{ }^{\circ} \mathrm{C}$ of UM silica was due to the dehydroxylation and that of the other two modified/washed and unwashed silica fillers are due to the dehydroxylation and polymer degradation. The polymer totally decomposes by heating up to $600^{\circ} \mathrm{C}$ as in Figure 3(a). Therefore, the percentage of chemically and physically bound polymer is found as 2.04 and $4.98 \%$ in MFnW and MFn, respectively after eliminating the percentage of weight corresponding to dehydroxylation. When the $\mathrm{pH}$ of aqueous dispersions of the fillers is increased, the surface attached polymer molecules have a tendency to dissolve due to the ionization of free or unreacted carboxylic acid groups. Therefore the polymer chains get expanded at $\mathrm{pH} 7$ as a result of anionic charge repulsion and increases the stability of the dispersion. However, by increasing the $\mathrm{pH}$ further up to 10 , the carboxylic groups may get totally ionized and simultaneously, the concentration of the counter ions $\left(\mathrm{K}^{+}\right)$may also increase. As a result of that the carboxylic anions formed are shielded by the $\mathrm{K}^{+}$ions minimizing the anionic charge repulsion and therefore, the polymer chains get contracted settling the MFa particles in the dispersion (Blacklley, 1997). However, the stability of the dispersion of MFn retains further up to $\mathrm{pH} 10$ as most of the carboxylic acid groups get involved in the formation of covalent bonds between the filler and the polymer. The remaining carboxylic acid groups may ionize but the concentration of counter ions may not be adequate to shield the anions created. Thus, the anionic charge on MFn may also increase the stability of filler dispersion by repulsing equally charged ions, which is electro-statistic stabilization. The stability of aqueous dispersion of UMF at all $\mathrm{pH}$ levels was very poor and the particle settling was observed.

After incorporation of the filler dispersions to NRL, it was observed that the UMF settled at the bottom of the container, however such a settling could not be observed in the modified filler added samples. The reason for this observation is that the interactions created between the filler and the polymer establish a compatibility between the rubber and the filler particles in latex, mainly by forming a network like structure as shown in Figure 8.

The microstructures of cross-sections of MF added NRL cast films also show an even dispersion confirming the compatibility of the modified filler with rubber in latex as shown in Figure 4 (c \& d). Figure 4(b) displays two separate layers of rubber and filler in the microstructure of the cross-section of UMF added NRL film. It shows the processing difficulties encountered in UMF incorporated NRL in large-scale to manufacture latex products (Hill, 2007).

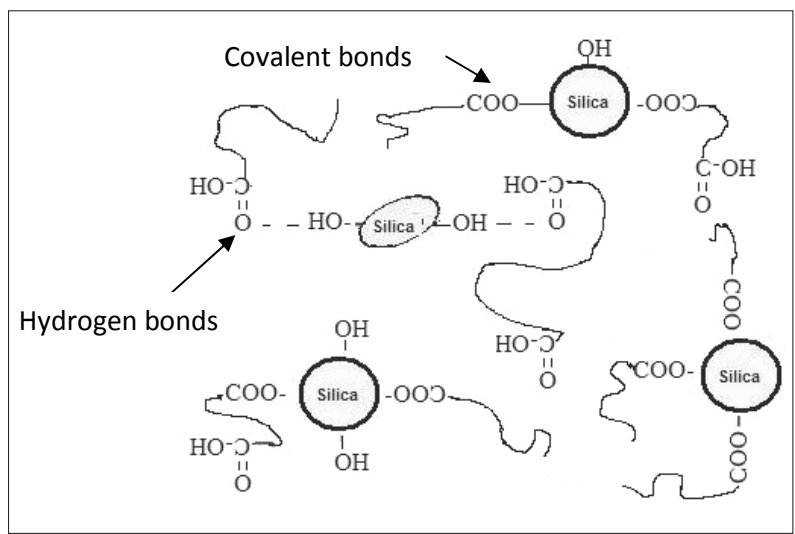

Figure 7: Schematic diagram of new bonds formed in between the filler and polymer

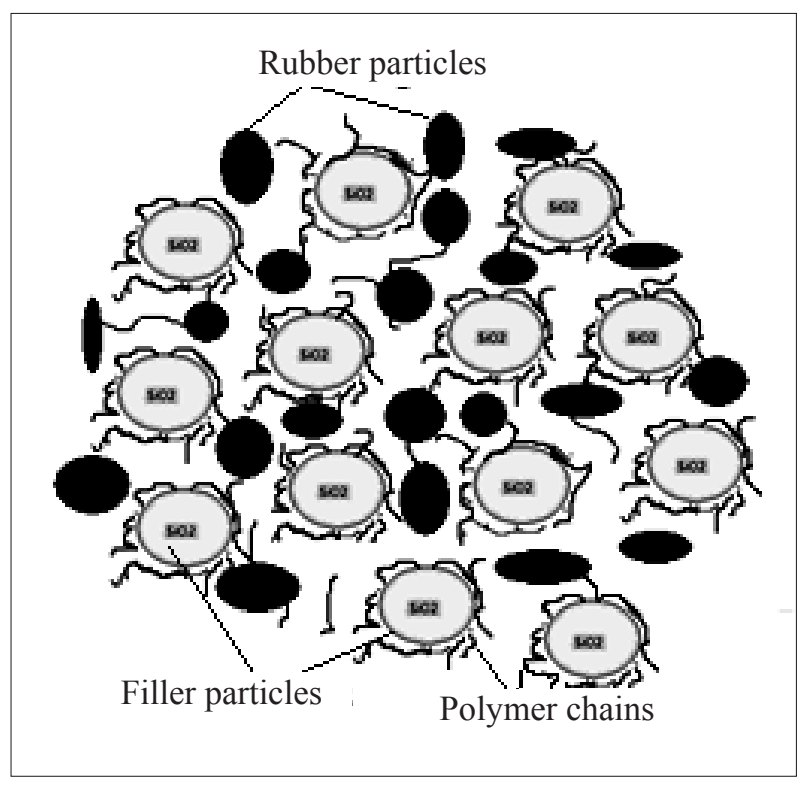

Figure 8: Schematic diagram of interactions created in between the modified-filler and rubber in latex 
The tear strength of all the films illustrated in Figure 5 increases at first, passes a maximum and then decreases with the increase of filler loadings. The maximum values of both modified filler added films are nearly $50 \%$ higher than that of UMF in the range of 5-10 phr of filler loadings. The tensile strength of the modified filler (MFa and MFn) added films (Figure 6) also shows a maximum at $5 \mathrm{phr}$ of filler addition, whilst that of UMF added films show a slight increment with the increase of filler concentration. However, the modified filler added films show nearly $35 \%$ increase at $5 \mathrm{phr}$ compared with the highest value of UMF added films at $20 \mathrm{phr}$. Accordingly the vulcanized films containing modified fillers show promising results of physical properties compared to that of the unfilled and UMF filler added films.

Uniform dispersion and distribution of the modified filler throughout the rubber matrix enhances the tear strength while additional tear strength is conferred by MFn because it has dispersed more evenly than MFa as observed in Figure 4(d). A promising tensile strength of rubber composite is also achieved by MFn; the reason may be the covalent bonds formed in between the filler and the polymer. The covalently bonded filler-polymer particles are strongly attached to the rubber phase, and as a result the stress applied on the composite transfers through rubber to the modified filler particles (Fu et al., 2008).

\section{CONCLUSION}

The surface of silica is successfully modified with low molecular weight hydrophilic polymer containing carboxylic acid groups. The polymer chains adsorbed chemically and physically to the surface of silica particles enhance the compatibility between silica and rubber increasing the dispersion and distribution of filler particles uniformly throughout the rubber matrix. Promising tear and tensile strengths were conferred to the latex vulcanizate at low concentrations of modified fillers and the improved mechanical properties envisage that the reinforcement of NRL films with surface modified silica.

\section{Acknowledgement}

The authors wish to thank the University Grants Commission for research funding.

\section{REFFERENCES}

1. Anasarifar A., Azhar A., Ibrahim N., Shaiah S.F. \& Lawton J.M.D. (2005). The use of silanised silica filler to reinforce and crosslink natural rubber. International Journal of Adhesion and Adhesive 25: 77 - 86.

DOI: http://dx.doi.org/10.1016/j.ijadhadh.2004.04.002

2. Azhgozhinova G.S., Güven O., Pekel N., Dubolazov A.V., Mun G.A. \& Nurkeeva Z.S. (2004). Complex formation of linear poly (methacrylic acid) with uranyl ions in aqueous solutions. Journal of Colloid and Interface Science 278: $155-159$.

DOI: http://dx.doi.org/10.1016/j.jcis.2004.05.010

3. Blacklley D.C. (1997). Application of latices. Polymer Latices Science and Engineering, volume 3, $2^{\text {nd }}$ edition, pp. 142 - 143. Chapman \& Hall, London, UK. DOI: http://dx.doi.org/10.1007/978-94-011-5848-0

4. Cai H., Li S., Tian G., Wang H. \& Wang J. (2003). Reinforcement of natural rubber latex film by ultra fine calcium carbonate. Journal of Applied Polymer Science 87: $952-985$.

DOI: http://dx.doi.org/10.1002/app.11410

5. Coates J. (2000). Interpretation of infrared spectra, a practical approach. Encyclopedia of Analytical Chemistry (ed. R.A. Meyers), pp. 10815 - 10837. John Wiley \& Sons Ltd., New York, USA.

6. Deng C., Chen M., Ao N., Yan D. \& Zheng Z. (2006). $\mathrm{CaCO}_{3} /$ natural rubber latex nanometer composite and its properties. Journal of Applied Polymer Science 101: 3442 $-3447$.

DOI: http://dx.doi.org/10.1002/app.24345

5. Fu S.Y., Feng X.Q., Lauke B. \& Mai Y.W. (2008). Effects of particle size, particle/matrix interface adhesion and particle loading on mechanical properties of particulate-polymer composites. Composites Part B 39: 933 - 961.

DOI: http://dx.doi.org/10.1016/j.compositesb.2008.01.002

6. Gultek A. \& Seckin T. (2002). Polymethacrylic acid and methacryloxypropyl trimethoxy silane /clay nanocomposites prepared by in-situ polymerization. Turk Journal of Chemistry 26: 925 - 937.

7. Hill D.M. (2007). Rubber latex films having improved tear resistance. United States Patent US2007/0254999A1

8. Huggins M.L. (1942). The viscosity of dilute solutions of long chain macromolecules dependence on concentration. American Chemical Society 64: 2716-2718.

9. International Organization for Standardization (2010). ISO 34-1:2010, Rubber, vulcanized or thermoplastic Determination of tear strength - Part 1: Trouser, angle and crescent test pieces. International Organization for Standardization, Geneva, Switzerland.

10. International Organization for Standardization (2011). ISO 37:2011, Rubber, vulcanized or thermoplastic - Determination of tensile stress - strain properties. International Organization for Standardization, Geneva, Switzerland.

11. Jeong D.S., Hong C.K., Lim G.T., Seo G. \& Seok C. (2009). Network silica with exceptional reinforcing performance for SBR compounds: interconnected by methylene diphenyl diisocianate. Elastomers and Plastics 41: 353. DOI: http://dx.doi.org/10.1177/0095244309103662

12. Manroshan S. \& Baharin A. (2005). Effect of nanosized calcium carbonate on the mechanical properties of 
latex films. Journal of Applied Polymer Science 96: $1550-1556$.

DOI: http://dx.doi.org/10.1002/app.21595

13. Mason J.C. (1975). Polymer Hand Book. John Wiely \& Sons Ltd., New York, USA.

14. Musići S., Filipović-Vincekovići N. \& Sekovanićii L. (2011). Precipitation of amorphous $\mathrm{SiO}_{2}$ particles and their properties. Brazilian Journal of Chemical Engineering 28: $89-94$.

15. Nah C., Huh M.Y., Rhee J.M. \& Yoon T.H. (2002). Plasma surface modification of silica and its effect on properties of styrene-butadiene rubber compound. Polymer International 51: $510-518$.

DOI: http://dx.doi.org/10.1002/pi.897

16. Nguyen V., Yoshida W. \& Cohen Y. (2003). Graft polymerization of vinyl acitate on silica. Journal of Applied Polymer Science 87: 300 - 310.

DOI: http://dx.doi.org/10.1002/app.11376

17. Nontasorn P., Chavadej S., Rangsunnvigit P., O'haver J.H., Chaisirimahamorakot S. \& Na-ranong N. (2005). Admicellar polymerization modified silica via a continuous stirred-tank reactor system: comparative properties of rubber compounding. Chemical Engineering 108: 213 - 218. DOI: http://dx.doi.org/10.1016/j.cej.2005.02.016

18. Park J.T., Seo J.A., Ahn S.H., Kim J.H. \& Kang S.W. (2010). Surface modification of silica nanoparticles with hydrophilic polymers. Journal of Engineering and Chemistry 16: $517-522$.

19. Peng Z., Kong L.X., Si-dong-li, Cheng Y. \& Huang M.F. (2007). Self assembled natural rubber/ silica nanocomposites: its preparation and characterization. Composite Science and Technology 67: 3130 - 3139.

DOI: http://dx.doi.org/10.1016/j.compscitech.2007.04.016

20. Prasertsri S. \& Rattanasom N. (2011). Mechanical and damping properties of silica/natural rubber composites prepared from latex system. Polymer Testing 30: $515-526$.

DOI: http://dx.doi.org/10.1016/j.polymertesting.2011.04.001

21. Rangsunvigit P., Imsawatgul P., Na-ranong N., O'haver J.H. \& Chavade S. (2008). Mixed surfactants for silica modification by admicellar polymerization using a continiuous stirred tank reactor. Chemical Engineering 136: $288-294$.

DOI: http://dx.doi.org/10.1016/j.cej.2007.03.087

22. Ru X., Yuchuan Z. \& Qingren Z. (2006). Study on nanoSi3N4/NBR composites'properties, Proceedings of the $5^{\text {th }}$ China International Nanoscience and Technology Xi'an Symposium, Xi'an, China, 10 - 13 September.
23. Salarizadeh P., Abdollahi M. \& Javanbakht M. (2012). Modification of silica nanoparticles with hydrophilic sulfonated polymers by using surface-initiated redox polymerization. Iranian Polymer Journal 21: 661 - 668 . DOI: http://dx.doi.org/10.1007/s13726-012-0073-7

24. Shing S.H. \& Kim H.I. (2001). Contribution of hydrogen bond and coupling reaction to improvement in compatibility of organic polymer/silica nanocomposites. Journal of Industrial and Engineering Chemistry 7: 147 - 152.

25. Suzuki K., Siddiqui S., Chappel C., Siddiqui J.A. \& Offenbrite R.M. (2000). Modification of porus silica paticles with poly (acrylic acid). Polymers for Advanced Technologies 11: $92-97$.

26. Tai Y., Qian J. \& Zhang Y. (2008). Study of surface modification of nano- $\mathrm{SiO}_{2}$ with macromolecular coupling agent. Chemical Engineering 141: $354-361$.

DOI: http://dx.doi.org/10.1016/j.cej.2008.03.012

27. Tian M., Liang W., Rao G., Zhang L. \& Guo C. (2005). Surface modification of fibrillar silicate and its reinforcing mechanism on FS/ rubber composites. Composite Science and Technology 65: $1129-1138$.

DOI: http://dx.doi.org/10.1016/j.compscitech.2004.11.008

28. Wanger M.P. (1976). Reinforcing silicas and silicates. Rubber Chemistry and Technology 49: 703.

DOI: http://dx.doi.org/10.5254/1.3534979

29. Wu C.S. \& Liao H.T. (2003). Polyethylene-octene elastomer/silica hybrids prepared by a sol-gel process using tetraethoxysilane. Designed Monomers and Polymers 6: $369-381$.

30. Xia R., Li M., Zhang Y., Zhu Q. \& Qian J. (2008a). Synthesis of tercopolymer BA-MMAVTES and surface modification of nano-size $\mathrm{Si}_{3} \mathrm{~N}_{4}$ with this macromolecular coupling agent. Applied Polymer Science 107: 1100 - 1107. DOI: http://dx.doi.org/10.1002/app.27175

31. Xia R., Zhang Y., Zhu Q., Qian J., Dong Q. \& Li F. (2008b). Surface Modification of nano-sized silicon nitride with BAMAA-AN tercopolymer. Applied Polymer Science 107: $562-570$.

DOI: http://dx.doi.org/10.1002/app.26563

32. Young R.P. (1969). Infrared spectroscopic studies of adsorption and catalysis. part 3 carboxylic acids and their derivatives adsorbed on silica. Canadian Journal of Chemistry 47: 2217 - 2247.

DOI: http://dx.doi.org/10.1139/v69-362

33. Zhuravlev L.T. (2000). The surface chemistry of amorphous silica, Zhuravlev model. Colloids and Surfaces A: Physicochemical and Engineering Aspects 173: 1 - 38. DOI: http://dx.doi.org/10.1016/S0927-7757(00)0055 6-2 